\title{
Programmable entanglement oscillations in a non-Markovian channel
}

\author{
Simone Cialdi, ${ }^{1,2, *}$ Davide Brivio,,${ }^{1, \dagger}$ Enrico Tesio,,${ }^{1, \ddagger}$ and Matteo G. A. Paris ${ }^{1, \S}$ \\ ${ }^{1}$ Dipartimento di Fisica dell'Università degli Studi di Milano, I-20133 Milan, Italy \\ ${ }^{2}$ INFN, Sezione di Milano, I-20133 Milan, Italy \\ (Received 22 September 2010; published 8 April 2011)
}

\begin{abstract}
We suggest and demonstrate an all-optical experimental setup to observe and engineer entanglement oscillations of a pair of polarization qubits in an effective non-Markovian channel. We generate entangled photon pairs by spontaneous parametric down-conversion (SPDC), and then insert a programmable spatial light modulator in order to impose a polarization-dependent phase shift on the spatial domain of the SPDC output. This creates an effective programmable non-Markovian environment where modulation of the environment spectrum is obtained by inserting a spatial grating on the signal arm. In our experiment, programmable oscillations of entanglement are achieved, where the entangled state obtained at the maximum of the revival after sudden death violates Bell's inequality by 17 standard deviations.
\end{abstract}

DOI: 10.1103/PhysRevA.83.042308

PACS number(s): 03.67.Bg, 03.65.Ud, 03.65.Yz, 42.50.Dv

\section{INTRODUCTION}

Entanglement of a bipartite system is usually degraded by the interaction of each subsystem with the environment, which induces decoherence, i.e., an irreversible loss of information from the system to the rest of the universe [1,2]. If the interaction is Markovian, i.e., the loss of information is unidirectional, from the system to the environment, then entanglement monotonically decreases and may be also destroyed in a finite time [3-7]. On the other hand, when some memory effect is present in the interaction between the system and the environment, i.e., when the noisy channel is non-Markovian [8,9], then a nonmonotone behavior of entanglement and, more generally, of quantum correlations may be observed [10-17]. In fact, entanglement oscillations are expected in continuous variable systems [18-20], whereas collapses and revivals of entanglement have been observed with polarization qubits [21].

In this paper we suggest and demonstrate an experimental setup to observe and engineer entanglement oscillations in a programmable way. We address the spatial domain of spontaneous parametric down-conversion (SPDC), and exploit a programmable spatial light modulator (SLM) to impose a polarization- and position-dependent phase shift. Since the polarization qubits are obtained by tracing out the spatial degrees of freedom, our apparatus allows us to analyze the entanglement dynamics within the "coherence time" of the effective non-Markovian channel. In this framework an effective environment spectrum may be obtained by acting on the spatial profile of the SPDC and, in particular, by a suitable modulation of the angular distribution. In turn, in order to investigate entanglement oscillations we insert a spatial grating on the signal arm to achieve a modulation of the environment spatial spectrum. Besides fundamental interest, our scheme may found applications in engineering decoherence for the polarization qubit, e.g., in quantum process tomography.

\footnotetext{
*simone.cialdi@mi.infn.it

†davide.brivio@unimi.it

†enrico.tesio@gmail.com

§matteo.paris@ fisica.unimi.it
}

The paper is structured as follows. In the next section we describe our scheme for entanglement generation and purification with a detailed analysis of the structure of the angular function. In Sec. III we describe how a periodic structure of the angular distribution may be used to induce entanglement oscillations in a programmable way, whereas entanglement decrease and then death may be expected for a nonperiodical angular distribution. Experimental results are described in detail in Sec. IV, with the full characterization of the involved states by quantum tomography reported in Sec. V. Section VI closes the paper with some concluding remarks.

\section{ENTANGLEMENT GENERATION AND PURIFICATION}

In our setup a two-crystal geometry [22-24] is used to produce two-qubit polarization entangled states by type I down-conversion in a noncollinear configuration. The state at the output of the crystal can be written as

$|\psi\rangle \propto \iint d \theta d \theta^{\prime} f\left(\theta, \theta^{\prime}\right)\left[|H \theta\rangle\left|H \theta^{\prime}\right\rangle+e^{\imath \Phi\left(\theta, \theta^{\prime}\right)}|V \theta\rangle\left|V \theta^{\prime}\right\rangle\right]$,

where $|P \theta\rangle$ denotes a single photon state emitted with polarization $P=H, V$ at angle $\theta\left(\theta^{\prime}\right)$ along the signal (idler) arm, $\theta$ and $\theta^{\prime}$ are the shifts from the central emission angle $\left(\theta_{0}, \theta_{0}^{\prime} \simeq 3^{\circ}\right)$, and the integrations range from $-\frac{1}{2} \Delta$ to $\frac{1}{2} \Delta$, $\Delta$ being the angular aperture of two slits along the downconversion paths; see Fig. 1.

The angle-dependent phase shift

$$
\Phi\left(\theta, \theta^{\prime}\right)=\phi(\theta)+\phi^{\prime}\left(\theta^{\prime}\right)+\Phi_{0}
$$

comes from the difference between the optical path of the vertically polarized photon pair, generated in the first crystal, which must travel along the second one, and the optical path of the pump beam traversing the first crystal before generating the horizontally polarized pair in the second crystal. These angular-dependent terms are responsible for decoherence of the polarization qubit and should be removed in order to obtain an effective source of entangled pairs [25]. Upon expanding to first order the terms in $\Phi\left(\theta, \theta^{\prime}\right)[26]$, we arrive at

$$
\phi(\theta)=\gamma \theta, \quad \phi^{\prime}\left(\theta^{\prime}\right)=-\gamma \theta^{\prime} .
$$




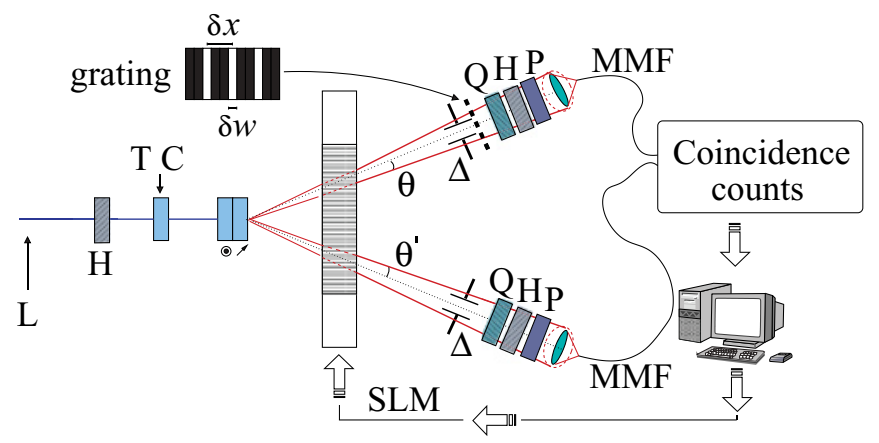

FIG. 1. (Color online) Schematic diagram of experimental setup. A linearly polarized $\mathrm{cw}$ laser diode at $406 \mathrm{~nm}(L)$ pumps a couple of BBO crystals cut for type I down-conversion. The horizontal and vertical photon pairs are balanced by a half-wave plate set along the pump path, whereas an additional BBO crystal is set on the pump path to compensate for the temporal delay. Signal and idler cones travel through the SLM and are spatially selected by two irises and two slits set at $D=500 \mathrm{~mm}$ with $\Delta x=5 \mathrm{~mm}(\Delta=10 \mathrm{mrad})$. Two long-pass filters cut-on wavelength $715 \mathrm{~nm}$ are used to reduce the background. A handmade grating can be inserted on the signal arm. Photons are focused in two multimode fibers (MMF's) and sent to single-photon counting modules. Polarizers at the angles $45^{\circ},-45^{\circ}$ or $45^{\circ}, 45^{\circ}$ are inserted to measure visibility whereas a quarter-wave plate, a half-wave plate, and a polarizer are used for the tomographic reconstruction.

In our apparatus, a one-dimensional programmable SLM is set both on the signal and idler paths (see Fig. 1), and is used to achieve a complete purification (i.e., $\Phi=\Phi_{0}$ ) by inserting a linear phase function $\phi_{\mathrm{SLM}}(\theta)=-\gamma \theta$ on the signal path and $\phi_{\mathrm{SLM}}^{\prime}\left(\theta^{\prime}\right)=\gamma \theta^{\prime}$ on the idler path [26-30]. The constant phase $\Phi_{0}$ allows the generation of different maximally entangled states.

\section{A. More details on the angular function}

In the present experiment we set $\Phi_{0}=0$ by adding a proper constant phase to $\phi_{S L M}$. In order to obtain an effective non-Markovian channel the SLM is then used to impose an additional phase function $\phi_{s}(\theta)$ on the signal arm. Before going to details, let us devote some attention to the angular function $f\left(\theta, \theta^{\prime}\right)$, which will be important for the following discussion and is assumed to have the factorized form $g(\theta) g^{\prime}\left(\theta^{\prime}\right)$. This assumption has been experimentally verified by measuring the coincidence count distribution $C=\left|f_{\text {expt }}\left(\theta, \theta^{\prime}\right)\right|^{2}$, within a coincidence time window of $50 \mathrm{~ns}$, as a function of the signal and idler slit positions $\theta$ and $\theta^{\prime}$. We set two slits of aperture $\Delta x=1 \mathrm{~mm}(\Delta=2 \mathrm{mrad})$ along the down-conversion arms and measured coincidence counts within a time window of $10 \mathrm{~s}$ for slit positions $\theta, \theta^{\prime}=-2 \Delta,-\Delta, \ldots,+2 \Delta$. In Fig. 2 we show the experimental data; the phase-matching central angles correspond to $\theta, \theta^{\prime}=0$. The corresponding coincidence count distribution has then been compared with the one computed as

$$
\frac{\left|f_{\text {expt }}(\theta, 0) f_{\text {expt }}\left(0, \theta^{\prime}\right)\right|^{2}}{\left|f_{\text {expt }}(0,0)\right|^{2}},
$$

and an excellent agreement was found, also corroborated by a significant $\chi^{2}$ test $\left(P_{\chi^{2}>\chi_{0}^{2}} \simeq 0.9\right)$. Upon inspecting the

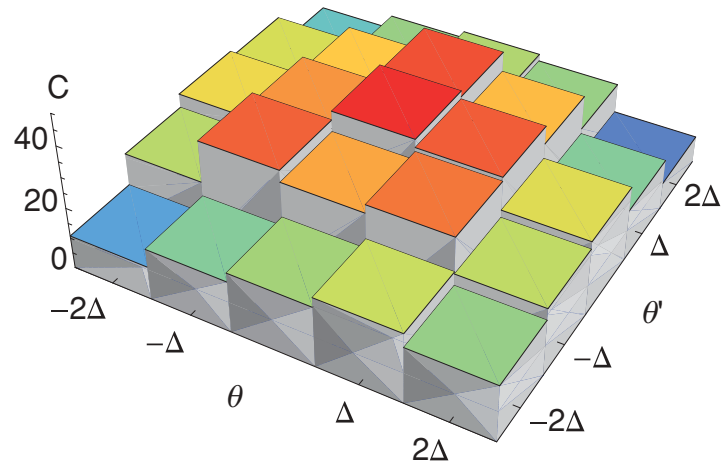

FIG. 2. (Color online) Coincidence count distribution $C=$ $\left|f_{\text {expt }}\left(\theta, \theta^{\prime}\right)\right|^{2}$ for $10 \mathrm{~s}$, within a coincidence time window of $50 \mathrm{~ns}$, as a function of the signal and idler slit positions $\theta$ and $\theta^{\prime}$. The phase-matching central angles correspond to $\theta, \theta^{\prime}=0$.

distribution of Fig. 2 one sees that the angular distributions $g(\theta), g^{\prime}\left(\theta^{\prime}\right)$ are peaked around the central values $\theta=\theta^{\prime}=0$ value with a width of $8.6 \mathrm{mrad}$. They may be approximated by Gaussian curves, though the specific functional form is irrelevant for our analysis. As will be clear in the following, factorization simplifies the evaluation of visibility.

\section{ENTANGLEMENT OSCILLATIONS IN A NON-MARKOVIAN ENVIRONMENT}

Once the state has been purified and the additional phase $\phi_{s}(\theta)=\alpha \theta$ has been imposed to the signal photon, the dependence on $\theta^{\prime}$ is traced out, and thus no terms containing $g^{\prime}\left(\theta^{\prime}\right)$ appear in the polarization density matrix,

$$
\begin{aligned}
\varrho= & \operatorname{Tr}_{\theta, \theta^{\prime}}[|\psi\rangle\langle\psi|]=\frac{1}{2}(|H H\rangle\langle H H|+\varepsilon| V V\rangle\langle H H| \\
& \left.+\varepsilon^{*}|H H\rangle\langle V V|+| V V\rangle\langle V V|\right),
\end{aligned}
$$

where

$$
\varepsilon=\int d \theta|g(\theta)|^{2} e^{\imath \alpha \theta}
$$

is the decoherence factor. It can be shown that, for the state $\varrho$, the concurrence is $\mathcal{C}=|\varepsilon|$ [21]. Since the angular distribution $g(\theta)$ is symmetric, $\varepsilon$ is real and positive, and we may write

$$
\varrho=\varepsilon \varrho_{b}+(1-\varepsilon) \varrho_{m},
$$

where $\varrho_{b}$ denotes a Bell state and $\varrho_{m}$ the corresponding mixture. In turn, in this case, $\varepsilon$ equals the interferometric visibility $V(\alpha)=\operatorname{Re}[\varepsilon]$ which, in turn, coincides with the concurrence $\mathcal{C}$.

In general, the angular distribution may be assumed to be peaked at some central value with a finite width as, for example, the Gaussian shape we have discussed in the preceding section. In this case, the photons coming from different directions (i.e., angles) will experience different relative phase between the horizontal and vertical polarization components. As a consequence, in the absence of any purification mechanism, the off-diagonal elements of the polarization density matrix decrease exponentially and the state is driven toward the unentangled mixture $\varrho_{m}$. 
The situation changes dramatically if the angular distribution is amplitude modulated. In this case the decoherence factor $\varepsilon$ may oscillate, i.e., the overall relative phase may refocus and the off-diagonal elements reappear, as well as entanglement. Since we address the spatial domain, it is straightforward to insert an amplitude modulation on $g(\theta)$, e.g., by inserting a physical obstacle along the signal optical path. As noted above, from the expression of the visibility $V(\alpha)$, we see that a periodic structure of the angular distribution would induce oscillations, whereas entanglement decrease and then death may be expected for a nonperiodical angular distribution. In this framework $\alpha$ may be considered as the evolution parameter of the dynamics of the (noisy) channel, and the periodic structure of the angular distribution as the (spatial) enviroment spectrum. Moreover, the periodic structure implies that in tracing out the spatial degrees of freedom any two-point correlation function of the environment is not single peaked and this is a sign of the non-Markovian character of the channel.

In our apparatus, the amplitude modulation is implemented by means of a handmade grating with a period $\delta x$ and a transparent region width $\delta w$ centered along the signal arm (see Fig. 1). As we will see, the narrower the transparent regions, the higher entanglement oscillations are expected. Formally, the insertion of the grating is equivalent to the substitution

$$
g(\theta) \rightarrow g(\theta) m(\theta)
$$

in the visibility, up to the normalization $\int d \theta|g(\theta) m(\theta)|^{2}=1$, where $m(\theta)$ is the periodical unitary step function imposed by the grating. By simply inserting or removing the grating it is possible to compare the different dynamics imposed by a periodical or nonperiodical angular distribution.

\section{EXPERIMENTAL RESULTS}

The experimental setup is shown in Fig. 1: a linearly polarized cw, 406-nm laser diode (Thorlabs LPS-406-FC), with a transverse profile $\mathrm{TEM}_{00}$, pumps a couple of 1-mm-thick $\beta$-barium borate (BBO) crystals cut for type I downconversion. The $|H H\rangle$ and $|V V\rangle$ pairs are balanced by a half-wave plate set along the pump path. A BBO crystal with the proper length and optical axis angle is set on the pump path, and is used to counteract the decoherence effect due to the temporal delay between the two components [25-35]. This crystal introduces a delay time between the horizontal and vertical polarization of the pump which precompensates for the delay time between the $|V V\rangle$ pair generated by the first crystal and the $|H H\rangle$ pair from the second one. Signal and idler cones then travel through the SLM and are spatially selected by two irises and two slits set at $D=500 \mathrm{~mm}$. The low quantum efficiency of our detectors $(\sim 10 \%)$ forces us to couple large angular regions: we set $\Delta x=5 \mathrm{~mm}(\Delta=10 \mathrm{mrad})$. As we discuss in the following, this will decrease the maximum value of the visibility. The down-conversion output is not spectrally filtered, whereas two long-pass filters (cut-on wavelength $715 \mathrm{~nm}$ ) are used to reduce the background. A handmade grating can be inserted on the signal arm.
Photons are focused in two multimode fibers and sent to homemade single-photon counting modules, based on an avalanche photodiode operated in Geiger mode with passive quenching. In order to measure the visibility, we insert two polarizers, set at the angles $45^{\circ},-45^{\circ}$ for the minimum and $45^{\circ}, 45^{\circ}$ for the maximum. For the tomographic reconstruction we insert on both paths a quarter-wave plate, a half-wave plate, and a polarizer.

After purification we study the behavior of the visibility as a function of the dimensionless evolution parameter $\alpha$, governing the linear phase function $\phi_{s}(\theta)=\alpha \theta$ imposed to the signal by the SLM. As previously discussed, oscillations of entanglement are expected when the grating is inserted. Because of the pixel discretization a step function with an angular resolution $\zeta=0.3 \mathrm{mrad}$ is physically inserted by the SLM in order to approximate the linear functions $\phi_{\mathrm{SLM}}(\theta), \phi_{\mathrm{SLM}}^{\prime}\left(\theta^{\prime}\right)$. Experimentally, using the SLM, we impose the phase functions

$$
\begin{array}{ll}
\phi_{\mathrm{SLM}}^{e}(n)=-a_{\mathrm{opt}} n+b & \text { on idler, } \\
\phi_{\mathrm{SLM}}^{e}(n)=a_{\mathrm{opt}} n+\phi_{s}^{e}(n) & \text { on signal, }
\end{array}
$$

where $n$ is the distance in pixels from the center of the signal beam ( $n=0$ for $\theta=0), a_{\text {opt }}=0.12 \mathrm{rad} / \mathrm{pixel}$ is the optimal slope that allows us to achieve a complete purification, and $b=$ $-\Phi_{0}$. The linear function $\phi_{s}^{e}(n)=a n$ is also inserted to study the dynamics, where the experimental evolution parameter is given by $a=\alpha h / L \mathrm{rad} /$ pixel, $h=100 \mu \mathrm{m}$ being the pixel width and $L \simeq 330 \mathrm{~mm}$ the distance between the SLM and the generating crystals. Since the pixel discretization of the SLM imposes the condition $a \ll 2 \pi /$ pixel, high values of $a$ must be neglected in our analysis. We experimentally verified that the curve $V(a)$ saturates to the uncompensated value when $\left(a+a_{\mathrm{opt}}\right) \rightarrow 2 \pi$. The revival is expected at

$$
a_{\mathrm{rev}}=\frac{2 \pi D}{\delta x} \frac{h}{L}
$$

or, in terms of the angular grating period $\delta \theta, \alpha_{\mathrm{rev}}=\frac{2 \pi}{\delta \theta}$. We choose $\delta x=2 \mathrm{~mm}$, which leads at $a_{\mathrm{rev}}=0.476 \mathrm{rad} / \mathrm{pixel}$, in order to avoid high values of the evolution parameter, and we set $\delta w=0.4 \delta x$.

In Fig. 3 we present the experimental results, together with the theoretical prediction calculated from the expression

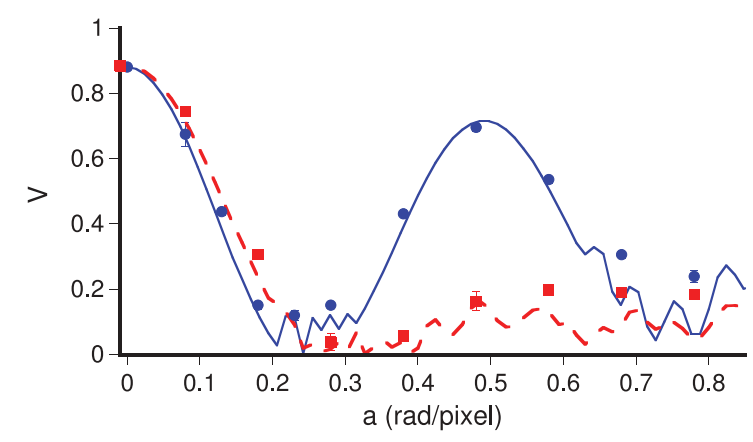

FIG. 3. (Color online) Visibility as a function of the evolution parameter $a$. Blue circles and red squares are the experimental data obtained with and without grating (errors within the symbols). The blue solid line and the red dashed line denote the corresponding theoretical predictions. 
of the visibility, as a function of the experimental evolution parameter $a$. Notice that the area under the increasing part of the visibility curve provides an experimental lower bound to the amount of non-Markovianity of the channels $[8,36]$.

Comparing the curve with the one obtained without the grating we see that in the latter case no revival occurs after the degradation of entanglement. We also notice that the minimum occurs for lower values of $a$ compared to the case with the grating.

\section{STATE CHARACTERIZATION}

In order to fully characterize the output state we have also performed state reconstruction by polarization qubit tomography for different values of the evolution parameter $a$. The procedure is as follows: we measure a suitable set of independent two-qubit projectors $[37,38]$ and then reconstruct the density matrix from the experimental probabilities using maximum-likelihood reconstruction of two-qubit states. The tomographic measurements are obtained by inserting a quarter-wave plate, a half-wave plate, and a polarizer. The purification procedure with the grating inserted leads to a visibility $V=0.881 \pm 0.004$, the density matrix is graphically represented in the upper left panel of Fig. 4. Increasing the evolution parameter to $a=0.23$, the visibility decreases to $V=0.120 \pm 0.016$. The corresponding tomographic
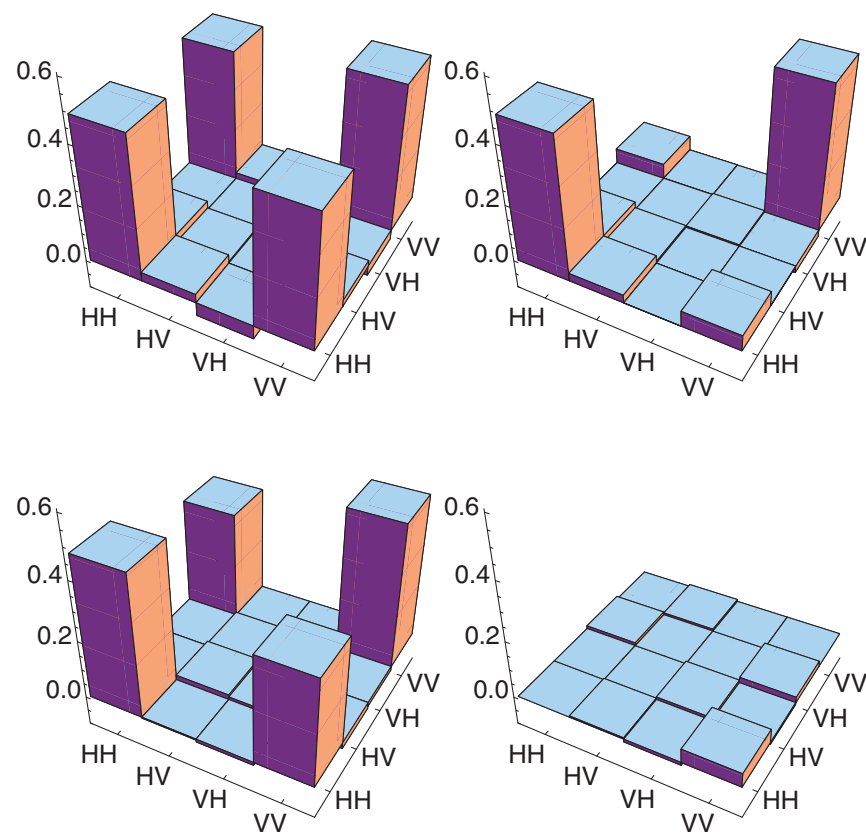

FIG. 4. (Color online) Tomographic reconstruction of a state evolving in the effective non-Markovian channel. In the upper left plot the two-qubit density matrix just after the purification, with visibility $V=0.881 \pm 0.004$. Upon increasing the evolution parameter to $a=0.23$ we achieve the minimum of entanglement oscillations: the density matrix is shown in the upper right plot, the corresponding visibility is $V=0.120 \pm 0.016$. In the lower panels, we show the real and the imaginary parts of the reconstructed density matrix at the maximum of entanglement oscillations, which occurs for $a=0.48$. The corresponding visibility is $V=0.696 \pm 0.013$, and the Bell parameter $B=2.341 \pm 0.019$. reconstruction, depicted in the upper right panel of Fig. 4, well illustrates the degradation of entanglement. However, we found a consistent revival after a further increasing of the evolution parameter to $a=0.48$, where we have $V=0.696 \pm$ 0.013. The corresponding tomographic reconstruction (real and imaginary parts) is reported in the lower panels of Fig. 4.

In order to show the revival of the nonlocal correlations we have also measured the Bell parameter,

$$
B=\left|E\left(\beta_{1}, \beta_{2}\right)+E\left(\beta_{1}, \beta_{2}^{\prime}\right)+E\left(\beta_{1}^{\prime}, \beta_{2}\right)-E\left(\beta_{1}^{\prime}, \beta_{2}^{\prime}\right)\right|,
$$

where $E\left(\beta_{1}, \beta_{2}\right)$ denotes the correlations between measurements performed at polarization angle $\beta_{j}$ for the mode $j$. We found $B=2.341 \pm 0.019$, which violates Clauser-HorneShimony-Holt-Bell inequality [39] by more than 17 standard deviations.

The residual lack of visibility after the purification procedure is mainly due to the very broad down-conversion spectral range that is selected by the slits. In fact, with the selected slit aperture, $\Delta x=5 \mathrm{~mm} \rightarrow \Delta=10 \mathrm{mrad}$, we estimate that about $200 \mathrm{~nm}$ of the down-conversion spectrum are coupled. By narrowing the slit aperture to $\Delta=4 \mathrm{mrad}$ only $60 \mathrm{~nm}$ are selected, and the visibility is found to increase at $V=$ $0.963 \pm 0.005$. The present experiment has been performed with the larger aperture to compensate for the low quantum efficiency of the photodetectors.

\section{CONCLUSIONS}

In conclusion, we have suggested and demonstrated an experimental setup to observe oscillations of polarization entanglement in a programmable way. Our scheme is based on a spatial light modulator, which is inserted on the spatial domain of the down-conversion output in order to impose a polarization-dependent phase shift on the spatial domain of the SPDC output. An effective programmable non-Markovian environment is then obtained by inserting a spatial grating on the signal arm. This results in a periodic structure of the angular distribution, which corresponds to modulation of the enviroment spectrum, and induces oscillations of entanglement, whereas entanglement decrease and then death is observed for a nonperiodical angular distribution.

In our experiment, programmable oscillations of entanglement are achieved by varying the linear phase function imposed to the signal by the SLM and verified both by visibility measurements and full polarization state tomography. The entangled state obtained at the maximum of the revival after sudden death violates Bell's inequality by 17 standard deviations.

Our scheme is all-optical and allows us to generate and detect revivals of entanglement and nonlocality, thus paving the way for engineering of decoherence for polarization qubits.

\section{ACKNOWLEDGMENTS}

M.G.A.P. thanks Sabrina Maniscalco, Stefano Olivares, Ruggero Vasile, Giuseppe Vallone, Marco Genovese, and Bassano Vacchini for useful discussions. 
[1] W. H. Zurek, Rev. Mod. Phys. 75, 715 (2003).

[2] M. Schlosshauer, Rev. Mod. Phys. 76, 1267 (2005).

[3] K. Zyczkowski, P. Horodecki, M. Horodecki, and R. Horodecki, Phys. Rev. A 65, 012101 (2001).

[4] T. Yu and J. H. Eberly, Phys. Rev. Lett. 93, 140404 (2004); Science 323, 598 (2009).

[5] M. P. Almeida, F. Melo, M. Hor-Meyll, A. Salles, S. P. Walborn, P. H. Souto Ribeiro, and L. Davidovich, Science 316, 579 (2007).

[6] J. Laurat, K. S. Choi, H. Deng, C. W. Chou, and H. J. Kimble, Phys. Rev. Lett. 99, 180504 (2007).

[7] J-S. Xu, X-Y. Xu, C-F. Li, C-J. Zhang, X-B. Zou, and G. Guo, Nature Commun. 1, 1 (2010).

[8] H-P. Breuer, E-M. Laine, and J. Piilo, Phys. Rev. Lett. 103, 210401 (2009).

[9] A. Rivas, S. F. Huelga, and M. B. Plenio, Phys. Rev. Lett. 105, 050403 (2010).

[10] M. Yonac and J. H. Eberly, Opt. Lett. 33, 270 (2008).

[11] S. Maniscalco, F. Francica, R. L. Zaffino, N. Lo Gullo, and F. Plastina, Phys. Rev. Lett. 100, 090503 (2008).

[12] L. Mazzola, S. Maniscalco, J. Piilo, K.-A. Suominen, and B. M. Garraway, Phys. Rev. A 79, 042302 (2009).

[13] B. Bellomo, R. Lo Franco, S. Maniscalco, and G. Compagno, Phys. Rev. A 78, 060302(R) (2008).

[14] K. Härkönen, F. Plastina, and S. Maniscalco, Phys. Rev. A 80, 033841 (2009).

[15] B. Bellomo, R. Lo Franco, and G. Compagno, Phys. Rev. Lett. 99, 160502 (2007); Adv. Sc. Lett. 2, 459 (2009).

[16] Q.-J. Tong, J.-H. An, H.-G. Luo, and C. H. Oh, Phys. Rev. A 81, 052330 (2010).

[17] F. F. Fanchini, T. Werlang, C. A. Brasil, L. G. E. Arruda, and A. O. Caldeira, Phys. Rev. A 81, 052107 (2010).

[18] S. Maniscalco, S. Olivares, and M. G. A. Paris, Phys. Rev. A 75, 062119 (2007).

[19] R. Vasile, S. Olivares, M. G. A. Paris, and S. Maniscalco, Phys. Rev. A 80, 062324 (2009).
[20] R. Vasile, P. Giorda, S. Olivares, M. G. A. Paris, and S. Maniscalco, Phys. Rev. A 82, 012313 (2010).

[21] J.-S. Xu, C.-F. Li, M. Gong, X.-B. Zou, C.-H. Shi, G. Chen, and G.-C. Guo, Phys. Rev. Lett. 104, 100502 (2010).

[22] L. Hardy, Phys. Lett. A 161, 326 (1992).

[23] P. G. Kwiat, E. Waks, A. G. White, I. Appelbaum, and P. H. Eberhard, Phys. Rev. A 60, R773 (1999).

[24] M. Genovese, Phys. Rep. 413, 319 (2005).

[25] R. Rangarajan, M. Goggin, and P. Kwiat, Opt. Express 17, 18920 (2009).

[26] S. Cialdi, D. Brivio, and M. G. A. Paris, Appl. Phys. Lett. 97, 041108 (2010)

[27] Z.-Q. Zhou, C.-F. Li, G. Chen, J.-S. Tang, Y. Zou, M. Gong, G.-C. Guo, Phys. Rev. A 81, 064302 (2010).

[28] S. Cialdi, D. Brivio, and M. G. A. Paris, Phys. Rev. A 81, 042322 (2010).

[29] S. Cialdi, F. Castelli, I. Boscolo, and M. G. A. Paris, Appl. Opt. 47, 1832 (2008).

[30] S. Cialdi, F. Castelli, and M. G. A. Paris, J. Mod. Opt. 56, 215 (2009).

[31] Y.-H. Kim, S. P. Kulik, and Y. Shih, Phys. Rev. A 62, 011802 (2000).

[32] Y. Nambu, K. Usami, Y. Tsuda, K. Matsumoto, and K. Nakamura, Phys. Rev. A 66, 033816 (2002).

[33] C. Cinelli, G. Di Nepi, F. De Martini, M. Barbieri, and P. Mataloni, Phys. Rev. A 70, 022321 (2004).

[34] M. Barbieri, C. Cinelli, F. De Martini, and P. Mataloni, Las. Phys. 16, 1439 (2006).

[35] G. Brida, M. Genovese, M. V. Chekhova, and L. A. Krivitsky, Phys. Rev. A 77, 015805 (2008).

[36] S. Cialdi, D. Brivio, E. Tesio, M. G. A. Paris (unpublished).

[37] K. Banaszek, G. M. D’ Ariano, M. G. A. Paris, and M. F. Sacchi, Phys. Rev. A 61, 010304(R) (1999).

[38] D. F. V. James, P. G. Kwiat, W. J. Munro, and A. G. White, Phys. Rev. A 64, 052312 (2001).

[39] J. F. Clauser, M. A. Horne, A. Shimony, and R. A. Holt, Phys. Rev. Lett. 23, 880 (1969). 